\title{
Rapid High-resolution Brain Mapping with CLARITY Optimized Light Sheet Microscopy (COLM)
}

\author{
Raju Tomer ${ }^{1,4}$, Karl Deisseroth ${ }^{1,2,3,4}$
}

${ }^{1}$ Department of Bioengineering, ${ }^{2}$ Department of Psychiatry and Behavioral Sciences, ${ }^{3}$ Howard Hughes Medical Institute, ${ }^{4} \mathrm{CNC}$ Program Stanford University, Stanford, CA

A major goal in neuroscience is to map the architecture of brain with both high wiring-level details and a brain-wide perspective. This challenge has drawn the attention of generations of scientists, while excellent progress has been made, many challenges and opportunities remain.

Electron Microscopy (EM) is the benchmark method for brain mapping studies [1-2]. EM provides a few nanometer imaging resolutions that allows reconstruction of the finest details of neural circuits. However, EM imaging speed is still generally slow (hence restricted to small blocks of tissues) and automated reconstruction has proven to be challenging. On the other end, MRI based technologies such as Diffusion Tensor Imaging (DTI) provides a quick view of the major neuronal fiber pathways in the brain. However, DTI generally lacks in resolution (about a millimeter) and it is still unclear what these tracts actually represent. There is an obvious disconnect in the high wiring level details of EM imaging and the brain-wide perspective of DTI. Light microscopy approaches, with sub-micron resolution and relatively fast imaging speed, can potentially bridge this gap. In addition, light microscopy combines well with the genetic labelling techniques allowing visualization of the sub-cellular details. One major limitation of light microscopy has been the opaqueness of brain and other biological tissue in the visible light spectrum. This has received increased attention over the last decade, resulting in many new chemical tissue clearing methods ([3-4], among others).

Our lab recently developed a new method called CLARITY [5-6] that provides excellent tissue clearing while preserving the molecular and structural content - a feature generally lacking in other methods. The basic idea of CLARITY is to build a highly cross-linked network of hydrogel inside the tissue. This is achieved first by infusion of a cocktail of formaldehyde and hydrogel monomer acrylamide into the tissue, followed by thermal initiation of the polymerization reaction inside the tissue resulting in a highly stable network. In the next step, all the cell membrane lipids are removed from the tissue, either by using electric field [5] or by a simple passive clearing protocol [6], resulting in a highly transparent stable tissue-hydrogel hybrid that is amenable to multiple rounds of specific histochemical labelling and imaging.

The next major challenge is to develop optimized high-speed and high-resolution microscopy methods to image large intact transparent tissue in entirety. To address this, we developed CLARITY Optimized Light-sheet Microscopy (COLM [6], Figure 1), building upon the 100 years old idea of light-sheet microscopy [7]. The basic idea of light-sheet microscopy is to illuminate the sample with a thin sheet of light and collecting the emitted fluorescence with an orthogonally arranged wide field detection arm. This optical decoupling of illumination and detection provides two key advantages over the point-scanning Confocal and 2-photon microscopy: (a) high imaging speed, owing to the use of fast sCMOS or CCD cameras, and (b) low photo-bleaching. The light-sheet microscopy idea has been revived in the past decade, 
largely in the context of developmental biology studies [8]. Additional optimizations were needed to achieve high-resolution deep imaging of large intact samples. COLM encompass three major innovations. Firstly, an optically homogeneous sample mounting approach was developed to minimize any optical aberrations. Secondly, synchronized illumination-detection strategy was employed to reduce the background signal. Finally, an adaptive parameter correction procedure was developed to achieve optimal image quality in the entire sample.

COLM enables several novel experimentations such as imaging of intact mouse brains, spinal cord, adult Zebrafish and large pieces of primate brains. Combined with genetic labelling methods, COLM has been used for cell types, structural and functional mapping of mouse and zebrafish brains. [9]

References:

[1] DD Bock et al. Nature 471 (2011), 177-182.

[2] KL Briggman, M Helmstaedter, W Denk, Nature 471 (2011), 183-188.

[3] HU Dodt et al. Nat. Methods 4 (2007), 331-336

[4] H Hama et al. Nat. Neuroscience 14 (2011), 1481-1488

[5] K Chung et al. Nature 497 (2013), 332-337.

[6] R Tomer et al. Nature protocols 9 (2014),1682-1697.

[7] H Siedentopf, R Zsigmondy, Annalen der Physik 10 (1903), 1-39.

[8] J Huisken, DY Stainier, Development 136 (2009), 1963-1975.

[9] We thank the entire Deisseroth lab for helpful discussions. All the tools and methods described are distributed and supported freely (clarityresourcecenter.org/COLM.html)

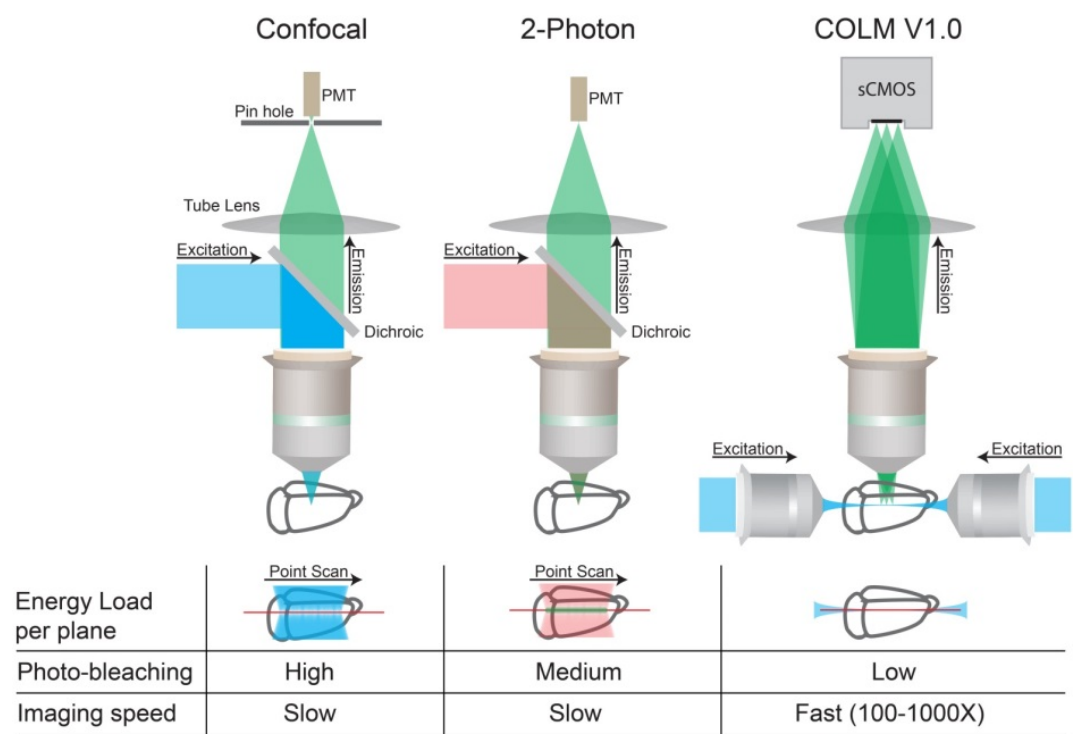

Figure 1. Comparison of confocal, two-photon and COLM. Confocal achieves optical sectioning by employing a pinhole. Two-photon utilizes the fact that only simultaneous absorption of two photons results in fluorescence signal. COLM, built upon light-sheet microscopy principles, achieves optical sectioning by confining the illumination to the plane of interest, and is several hundred times faster with minimal photo-bleaching. 